\title{
STARLIKENESS AND CONVEXITY FROM INTEGRAL MEANS OF THE DERIVATIVE
}

\author{
SHINJI YAMASHITA
}

(Communicated by Irwin Kra)

ABstract. If $f$ is analytic in $|z|<1$ and normalized: $f(0)=f^{\prime}(0)-1=0$, then $f$ is univalent and starlike in $|z|<I(f)$, where

$$
I(f)=\sup r\left\{(2 \pi)^{-1} \int_{0}^{2 \pi}\left|f^{\prime}\left(r e^{i t}\right)\right|^{2} d t\right\}^{-1 / 2}, \quad 0 \leq r<1 .
$$

Furthermore, there exists a normalized $f$ such that $I(f)<1$ and that $f^{\prime}$ vanishes at a point on $|z|=I(f)$.

If $f$ is analytic and normalized in $|z|<1$, then $f$ is univalent and convex in $|z|<I(f) / 2$.

1. Introduction. Let $F$ be the family of functions $f$ analytic in $D=\{|z|<1\}$ with $f(0)=f^{\prime}(0)-1=0$. The radius of starlikeness $\sigma(f)$ of $f \in F$ is the largest $r$ such that $f$ is univalent in $D(r)=\{|z|<r\}$ and $c f(z) \in f(D(r))$ for all $z \in D(r)$ and all $c, 0<c<1$, where $0<r \leq 1$. Setting

$$
M_{2}\left(r, f^{\prime}\right)=\left\{(2 \pi)^{-1} \int_{0}^{2 \pi}\left|f^{\prime}\left(r e^{i t}\right)\right|^{2} d t\right\}^{1 / 2}, \quad 0 \leq r<1
$$

and

$$
\Phi_{2}(f)=\sup _{0 \leq r<1} r / M_{2}\left(r, f^{\prime}\right) \quad \text { for } f \in F
$$

we begin with

THEOREM 1. $\sigma(f) \geq \Phi_{2}(f)$ for $f \in F$.

Set $\left\|f^{\prime}\right\|_{2}=\lim _{r \rightarrow 1} M_{2}\left(r, f^{\prime}\right) \leq+\infty$. Since

$$
\Phi_{2}(f) \geq\left\|f^{\prime}\right\|_{2}^{-1} \geq 0
$$

it follows that

$$
\sigma(f) \geq\left\|f^{\prime}\right\|_{2}^{-1} \text { for } f \in F,
$$

a known result [G1, Theorem 23, p. 187] (see also [Gd2, II, p. 95]).

However, the estimate (2) is of no value in case $\left\|f^{\prime}\right\|_{2}=+\infty$, while Theorem 1 remains available because $\Phi_{2}(f)>0$ for each $f \in F$.

We can construct $f \in F$ such that

$$
\sigma(f)=\Phi_{2}(f)>\left\|f^{\prime}\right\|_{2}^{-1}>0
$$

Received by the editors March 9, 1987 and, in revised form, May 19, 1987.

1980 Mathematics Subject Classification (1985 Revision). Primary 30C55; Secondary 30C45. 
2. Proof of Theorem 1. See [D1] for general references for the mean $M_{p}(r, h)$ and the norm $\|h\|_{p}$ of $h$ analytic in $D, 0<p<+\infty$, and $0 \leq r<1$; thus, $H^{p}=\left\{h ;\|h\|_{p}<+\infty\right\}$, the Hardy class.

For $f \in F$ we set

$$
\Phi_{p}(r, f)=r\left\{1+M_{p}\left(r, f^{\prime}-1\right)^{p}\right\}^{-1 / p}, \quad 0 \leq r<1,
$$

and

$$
\Phi_{p}(f)=\sup _{0 \leq r<1} \Phi_{p}(r, f), \quad 0<p<+\infty ;
$$

we note that $\Phi_{2}(r, f)=r / M_{2}\left(r, f^{\prime}\right)$, so that $\Phi_{2}(f)$ is the same as in Theorem 1 . Apparently, $\Phi_{p}(f) \geq\left(1+\left\|f^{\prime}-1\right\|_{p}^{p}\right)^{-1 / p}$. Theorem 1 is now the case $p=2$ in

THEOREM 2. $\sigma(f) \geq \Phi_{p}(f)$ for $f \in F(1 \leq p \leq 2)$.

Comments on Theorem 2 for $1 \leq p<2$ will be given in Remark 1 . For the proof of Theorem 2 we shall make use of two lemmas.

LEMMA 1. If $h(z)=\sum_{n=1}^{\infty} b_{n} z^{n} \in F$, and if $\sum_{n=2}^{\infty} n\left|b_{n}\right| \leq 1$, then $\sigma(h)=1$.

See [Gd1, Theorem 1; CK, Theorem 3; D2, p. 73 and Gd2, I, p. 128].

LEMMA 2 [D1, Theorem 6.1, p. 94]. If $h(z)=\sum_{n=0}^{\infty} b_{n} z^{n} \in H^{p}(1 \leq p \leq 2)$, then

$$
\left(\sum_{n=0}^{\infty}\left|b_{n}\right|^{q}\right)^{1 / q} \leq\|h\|_{p} \quad(1 / p+1 / q=1)
$$

where the left-hand side is $\sup _{n \geq 0}\left|b_{n}\right|$ if $p=1$.

To prove Theorem 2 we may suppose that

$$
f(z)=\sum_{n=1}^{\infty} a_{n} z^{n} \not \equiv z .
$$

For each fixed $r, 0<r<1$, we set $R=\Phi_{p}(r, f)$. Then, $0<R<r$, and for $h(z)=f^{\prime}(r z)-1$, Lemma 2 yields that

$$
\left\{\sum_{n=2}^{\infty}\left(n\left|a_{n}\right| r^{n-1}\right)^{q}\right\}^{1 / q} \leq\|h\|_{p}=M_{p}\left(r, f^{\prime}-1\right) .
$$

The Hölder inequality enables us to have

$$
\begin{aligned}
\sum_{n=2}^{\infty} n\left|a_{n}\right| R^{n-1} & =\sum_{n=2}^{\infty} n\left|a_{n}\right| r^{n-1}(R / r)^{n-1} \\
& \leq M_{p}\left(r, f^{\prime}-1\right)\left\{\sum_{n=2}^{\infty}(R / r)^{p n-p}\right\}^{1 / p}=1 .
\end{aligned}
$$

With the aid of Lemma 1 we obtain $\sigma(g)=1$ for $g(z)=R^{-1} f(R z) \in F$, whence $\sigma(f) \geq R$. Since $r$ is arbitrary this completes the proof of Theorem 2 .

To construct $f \in F$ with (3) we fix $r, 0<r<1$, and then we choose $A$ such that $r^{-1}<A<2^{-1}\left(r+r^{-3}\right)$. Then, $f$ is defined by

$$
f(z)=A^{2} z-\left(A^{2}-1\right) A r \log \{r A /(r A-z)\}
$$


this is analytic in $\{|z|<r A\}$. Since

$$
f^{\prime}(z)=(-A) \cdot \frac{z / r-1 / A}{1-z /(r A)}
$$

it follows that $M_{2}\left(r, f^{\prime}\right)=A$, and hence $\Phi_{2}(f) \geq r / A$. On the other hand, $\sigma(f) \leq$ $r / A$ because $f^{\prime}(r / A)=0$. We thus arrive at $\sigma(f)=\Phi_{2}(f)=r / A$. For the norm of $f^{\prime} \in H^{2}$ we have

$$
\begin{aligned}
\left\|f^{\prime}\right\|_{2}^{2} & \geq A^{2}\left(r^{-1}-A^{-1}\right)^{2}(2 \pi)^{-1} \int_{0}^{2 \pi}\left|1-(r A)^{-1} e^{i t}\right|^{-2} d t \\
& =A^{2}\left(r^{-1}-A^{-1}\right)^{2}\left\{1-(r A)^{-2}\right\}^{-1}>(A / r)^{2}
\end{aligned}
$$

Therefore, $f$ satisfies (3).

REMARK 1. The existence of $f \in F$ with $\sigma(f)=\Phi_{p}(f)>\left(1+\left\|f^{\prime}-1\right\|_{p}^{p}\right)^{-1 / p}>0$ $(1 \leq p<2)$ is unknown. Also, it seems not easy to compare $\Phi_{p}(r, f)$ with $\Phi_{2}(r, f)$ in case $p<2$. We observe this for $p=1$ : sometimes, $\Phi_{1}(r, f)<\Phi_{2}(r, f)$, and sometimes, $\Phi_{1}(r, f)>\Phi_{2}(r, f)$.

Given $r, 0<r<1$, we observe that $f(z)=z+2^{-1} r^{-1} z^{2} \in F$ satisfies $\Phi_{1}(r, f)<$ $\Phi_{2}(r, f)$ because

$$
1+M_{1}\left(r, f^{\prime}-1\right)=2>2^{1 / 2}=M_{2}\left(r, f^{\prime}\right) .
$$

On the other hand, let $0<r<1$. Then, the function $f(z)=r G\left(r^{-1} z\right)$, where

$$
G(z)=z+2^{-1} A z^{2}-3^{-1} A z^{3}, \quad A>4 \pi /\left(\pi^{2}-8\right),
$$

satisfies $\Phi_{1}(r, f)>\Phi_{2}(r, f)$. Actually,

$$
\begin{aligned}
1+M_{1}\left(r, f^{\prime}-1\right) & =1+\left\|G^{\prime}-1\right\|_{1}=4 \pi^{-1} A+1 \\
& <\left(1+2 A^{2}\right)^{1 / 2}=\left\|G^{\prime}\right\|_{2}=M_{2}\left(r, f^{\prime}\right),
\end{aligned}
$$

where we make use of $\int_{0}^{2 \pi}\left|1-e^{i t}\right| d t=8$.

3. Radius of convexity. The radius of convexity $\kappa(f)$ of $f \in F$ is the largest $r(0<r \leq 1)$ such that $f$ is univalent in $D(r)$ and $c f(z)+(1-c) f(w) \in f(D(r))$ for all $z, w \in D(r)$ and all $c, 0<c<1$.

THEOREM $1 \mathrm{C} . \quad \kappa(f) \geq \Phi_{2}(f) / 2$ for $f \in F$.

We have no information on the sharpness. Theorem $1 \mathrm{C}$ is actually the case $p=2$ in

THEOREM 2 C. $\kappa(f) \geq \Phi_{p}(f) / 2$ for $f \in F(1 \leq p \leq 2)$.

We follow the same lines as in the proof of Theorem 2, where, in this case, Lemma 1 is replaced by $1 / 2$.

LEMMA $1 \mathrm{C}$. If $h(z)=\sum_{n=1}^{\infty} b_{n} z^{n} \in F$, and if $\sum_{n=2}^{\infty} n\left|b_{n}\right| \leq 1$, then $\kappa(h) \geq$

The estimate is exact since $\kappa\left(h_{0}\right)=1 / 2$ for $h_{0}(z)=z-2^{-1} z^{2}$. The proof of Lemma $1 \mathrm{C}$ depends on the following lemma. 
LEMMA 3. (See, for example, [Gd1, Theorem 1].) If $h(z)=\sum_{n=1}^{\infty} b_{n} z^{n} \in F$, and if $\sum_{n=2}^{\infty} n^{2}\left|b_{n}\right| \leq 1$, then $\kappa(h)=1$.

Lemma $1 \mathrm{C}$ is an exercise in [D2, p. 73], and the proof is in a few lines which we shall give for completeness. For $g(z)=2 h(z / 2)=\sum_{n=1}^{\infty} c_{n} z^{n} \in F$ we have

$$
\sum_{n=2}^{\infty} n^{2}\left|c_{n}\right| \leq \sum_{n=2}^{\infty} n\left|b_{n}\right| \leq 1
$$

by $n 2^{-n+1} \leq 1(n \geq 2)$, so that $\kappa(g)=1$ by Lemma 3 , and hence $\kappa(h) \geq 1 / 2$.

REMARK 2. Since $\Phi_{2}\left(f_{n}\right)=\left(1+n^{2}\right)^{-1 / 2} \rightarrow 0$ as $n \rightarrow \infty$ for $f_{n}(z)=z+$ $2^{-1} n z^{2} \in F$, it follows that $\Phi_{2}(F)=0$, where

$$
\Phi_{2}\left(F_{1}\right)=\inf \left\{\Phi_{2}(f) ; f \in F_{1}\right\} \quad \text { for } F_{1} \subset F \text {. }
$$

For what subfamily $F_{1}$ of $F$ have we $\Phi_{2}\left(F_{1}\right)>0$ ? A typical example is the family $S$ of all $f \in F$ univalent in $D$. We have

$$
\Phi_{2}(S) \geq \sup _{0 \leq x<1}\{x / \phi(x)\}^{1 / 2} \equiv c=0.164 \ldots,
$$

where

$$
\phi(x)=(1-x)^{-5}\left(x^{3}+11 x^{2}+11 x+1\right) .
$$

Remembering the known constants [D2, pp. 44 and 98, Gd2, I, pp. 119 and 121], due to H. Grunsky and R. Nevanlinna:

$$
\begin{gathered}
c_{G}=\inf \{\sigma(f) ; f \in S\}=\tanh (\pi / 4)=0.6557 \ldots, \\
c_{N}=\inf \{\kappa(f) ; f \in S\}=2-\sqrt{3}<c_{G} / 2,
\end{gathered}
$$

we have by Theorem $1 \mathrm{C}$ the estimates

$$
c \leq \Phi_{2}(S) \leq 2 c_{N}<c_{G} .
$$

It would be interesting to fill the considerable gap between $c$ and $2 c_{N}=0.535 \ldots$

For the proof of (4) we make use of the de Branges theorem [B] that $\left|a_{n}\right| \leq n$ $(n \geq 2)$ for $f(z)=\sum_{n=1}^{\infty} a_{n} z^{n} \in S$. Setting $x=r^{2}$ for $0<r<1$ we obtain

$$
M_{2}\left(r, f^{\prime}\right)^{2}=\sum_{n=1}^{\infty} n^{2}\left|a_{n}\right|^{2} r^{2 n-2} \leq \sum_{n=1}^{\infty} n^{4} x^{n-1}=\phi(x)
$$

so that

$$
\Phi_{2}(f) \geq c=\left\{x_{0} / \phi\left(x_{0}\right)\right\}^{1 / 2} \text { for } x_{0}=0.84 \ldots
$$

REMARK 3. Theorem $1 \mathrm{C}$ also follows directly from Lemma 3. For $f(z)=$ $\sum_{n=1}^{\infty} a_{n} z^{n} \not \equiv z$ and for $0<r<1$, let $Q=Q(r, f)$ be the real root of the equation

$$
A\left\{(1+x) /(1-x)^{3}-1\right\}=1, \quad A=M_{2}\left(r, f^{\prime}\right)^{2}-1
$$

We shall soon observe that $0<Q<1$. Set

$$
\Psi(r, f)=r Q(r, f)^{1 / 2}, \quad 0<r<1 .
$$

Then, we can show that

$$
\kappa(f) \geq \sup _{0<r<1} \Psi(r, f)
$$


and furthermore,

$$
\Psi(r, f) \geq \Phi_{2}(r, f) / 2 \quad(0<r<1)
$$

whence Theorem $1 \mathrm{C}$ follows.

We consider the cubic curve $Y=X^{3}+B X-2 B$ in the $X Y$-plane, where $B=A /(1+A)$. The curve cuts the $X$-axis at the only one point

$$
X_{0}=B^{1 / 3}\left[\left\{1+\left(1+\frac{B}{27}\right)^{1 / 2}\right\}^{1 / 3}+\left\{1-\left(1+\frac{B}{27}\right)^{1 / 2}\right\}^{1 / 3}\right]
$$

and has the straight line

$$
Y=(B+3) X-2 B-2
$$

as the tangent at $X=1$; the tangent cuts the $X$-axis at $X_{1}=2(B+1) /(B+3)$. By an elementary analysis we have $0<X_{0}<X_{1}<1$. Now the solution $Q$ of (6) is given by $Q=1-X_{0}$, so that simple calculations show that

$$
4^{-1} M_{2}\left(r, f^{\prime}\right)^{-2} \leq 1-X_{1}<Q<1 .
$$

We thus have (8).

For the proof of (7) we first note that $0<R \equiv \Psi(r, f)<r$. By the Schwarz inequality we obtain

$$
\begin{aligned}
\left(\sum_{n=2}^{\infty} n^{2}\left|a_{n}\right| R^{n-1}\right)^{2} & =\left(\sum_{n=2}^{\infty} n^{2}\left|a_{n}\right| r^{n-1}(R / r)^{n-1}\right)^{2} \\
& \leq M_{2}\left(r, f^{\prime}-1\right)^{2}\left(\sum_{n=2}^{\infty} n^{2}(R / r)^{2 n-2}\right)=1
\end{aligned}
$$

by (6), so that $\kappa(g)=1$ for $g(z)=R^{-1} f(R z)$ by Lemma 3. We thus have $\kappa(f) \geq R$, and this completes the proof of (7).

ADDED IN PROOF TO REMARK 2. For the Koebe function $k \in S$ we have $c=\Phi_{2}(k) \geq \Phi_{2}(S)$. Therefore $\Phi_{2}(S)=c$.

\section{REFERENCES}

[B] Louis de Branges, A proof of the Bieberbach conjecture, Acta Math. 154 (1985), 137-152.

[CK] J. Clunie and F. R. Keogh, On starlike and convex schlicht functions, J. London Math. Soc. 35 (1960), 229-233.

[D1] P. L. Duren, Theory of $H^{p}$ spaces, Academic Press, New York, 1970.

[D2] _ Univalent functions, Springer-Verlag, Berlin and New York, 1983.

[Gd1] A. W. Goodman, Univalent functions and nonanalytic curves Proc. Amer. Math. Soc. 8 (1957), 598-601.

[Gd2] _ Univalent functions. I, II, Mariner, Tampa, 1983.

[G1] G. M. Goluzin (Г. М. Голузин), To the theory of univalent conformal mappings (К теории однолистных конформных преобразовании), Mat. Sb. $42: 2$ (1935), 169-190.

Department of Mathematics, TOKyo Metropolitan University, Fukasawa, SETAGAYA, TOKYO 158, JAPAN 